\title{
Efficacy and safety of left atrial appendage closure versus medical treatment in atrial fibrillation: a network meta-analysis from randomised trials
}

\author{
Shweta Sahay, ${ }^{1}$ Luis Nombela-Franco, ${ }^{1}$ Josep Rodes-Cabau, ${ }^{2}$ Pilar Jimenez-Quevedo, ${ }^{1}$ \\ Pablo Salinas, ${ }^{1}$ Corina Biagioni, ${ }^{1}$ Ivan Nuñez-Gil, ${ }^{1}$ Nieves Gonzalo, ${ }^{1}$ \\ Jose Alberto de Agustín, ${ }^{1}$ Maria del Trigo, ${ }^{1}$ Leopoldo Perez de Isla, ${ }^{1}$ \\ Antonio Fernández-Ortiz, ${ }^{1}$ Javier Escaned, ${ }^{1}$ Carlos Macaya ${ }^{1}$
}

\begin{abstract}
${ }^{1}$ Instituto Cardiovascular, Hospital Universitario Clínico San Carlos, Madrid, Spain ${ }^{2}$ Quebec Heart and Lung Institute, Quebec City, Quebec, Canada
\end{abstract}

\section{Correspondence to} Dr Luis Nombela-Franco, Instituto Cardiovascular, Hospital Universitario Clínico San Carlos, C/ Prof. Martin Lagos s/n., Madrid 28040, Spain;

luisnombela@yahoo.com

SS and LN-F contributed equally to this study.

Received 10 April 2016 Revised 31 July 2016 Accepted 5 August 2016 Published Online First 1 September 2016

\section{SLinked}

- http://dx.doi.org/10.1136/ heartjnl-2016-310255

\section{CrossMark}

\footnotetext{
To cite: Sahay S, NombelaFranco L, Rodes-Cabau J, et al. Heart 2017;103:139147
}

\section{ABSTRACT}

Background The effectiveness of vitamin $\mathrm{K}$ antagonist (VKA) versus placebo and antiplatelet therapy (APT) is well established for stroke prevention in atrial fibrillation (AF). Non-vitamin K antagonist oral anticoagulants (NOAC) are mostly superior to VKA in stroke and intracranial bleeding prevention. Recent randomised controlled trials (RCTs) suggested the non-inferiority of percutaneous left atrial appendage closure (LAAC) versus VKA. However, comparisons between LAAC versus placebo, APT or NOAC are lacking. The purpose of this network meta-analysis was to assess the efficacy and safety of LAAC compared with other strategies for stroke prevention in patients with AF.

Methods We pooled together all RCTs comparing warfarin with placebo, APT or NOAC in patients with AF using meta-analysis guidelines. Two major trials of LAAC were also included and a network meta-analysis was performed to compare the impact of LAAC on mortality, stroke/systemic embolism (SE) and major bleeding in relation to medical treatment.

Results The network meta-analysis included 19 RCTs with a total of 87831 patients with AF receiving anticoagulants, APT, placebo or LAAC. Indirect comparison with network meta-analysis using warfarin as the common comparator revealed efficacy benefit favouring LAAC as compared with placebo (mortality: HR $0.38,95 \% \mathrm{Cl} 0.22$ to $0.67, \mathrm{p}<0.001$; stroke/SE: HR $0.24,95 \% \mathrm{Cl} 0.11$ to $0.52, p<0.001)$ and APT (mortality: HR $0.58,95 \% \mathrm{Cl} 0.37$ to $0.91, p=0.0018$; stroke/SE: HR $0.44,95 \% \mathrm{Cl} 0.23$ to $0.86, p=0.017$ ) and similar to NOAC (mortality: HR $0.76,95 \% \mathrm{Cl} 0.50$ to 1.16, $p=0.211$; stroke/SE: HR $1.01,95 \% \mathrm{Cl} 0.53$ to 1.92, $p=0.969$ ). LAAC showed comparable rates of major bleeding when compared with placebo (HR 2.33, $95 \% \mathrm{Cl} 0.67$ to $8.09, p=0.183$ ), APT (HR $0.75,95 \% \mathrm{Cl}$ 0.30 to $1.88, p=0.542$ ) and NOAC (HR $0.80,95 \% \mathrm{Cl}$ 0.33 to $1.94, p=0.615$ ).

Conclusions The findings of this meta-analysis suggest that LAAC is superior to placebo and APT, and comparable to NOAC for preventing mortality and stroke or SE, with similar bleeding risk in patients with nonvalvular AF. However, these results should be interpreted with caution and more studies are needed to further substantiate this advantage, in view of the wide $\mathrm{Cls}$ with some variables in the current meta-analysis.

\section{INTRODUCTION}

Stroke prevention in the elderly patients with nonvalvular atrial fibrillation (AF) is challenging due to associated high morbidity and mortality. ${ }^{1}$ Although warfarin is highly effective in stroke and systemic embolism (SE) prevention, its use is limited by numerous food and drug interactions, narrow therapeutic range and increased bleeding risk. ${ }^{2}{ }^{3}$ Most of these disadvantages have been partially mitigated by non-vitamin $\mathrm{K}$ antagonist oral anticoagulants (NOAC), but the risk of gastrointestinal bleeding continues with some NOAC as compared with warfarin ${ }^{4}$ and is definitely higher than in the absence of any antithrombotic therapy.

Left atrial appendage closure (LAAC) with the Watchman device (Boston Scientific, Marlborough, Massachusetts, USA) has been recently approved in the USA as an alternative to warfarin in patients with non-valvular $\mathrm{AF}^{5}$ based on the results of two randomised trials. ${ }^{6}$ As it obviates the need for long-term anticoagulation; the risk of bleeding is expected to be less with LAAC, in addition to maintaining the benefit of reduced stroke risk. However, direct comparison between NOAC and LAAC is lacking. In Europe, LAAC has a role in patients with thromboembolic risk who refuse or cannot be managed with any form of anticoagulation in the long term, ${ }^{8}$ in whom antiplatelet therapy (APT) or no treatment is generally proposed. No direct comparison exists between LAAC and placebo or APT, and it is unlikely that a randomised controlled trial (RCT) will be performed in this setting. Thus, the aim of this analysis was to assess the efficacy (mortality and stroke/SE) and safety (major, intracranial and gastrointestinal bleeding) of LAAC as compared with medical prophylactic therapy for stroke prevention in patients with non-valvular AF.

\section{METHODS}

Objectives and study design

The population of interest included patients with non-valvular $\mathrm{AF}$ and the intervention was medical or percutaneous treatment for stroke prophylaxis assessed in RCT. Comparisons were performed between medical therapy (NOAC, APT or placebo) and percutaneous (LAAC) treatment, using vitamin $\mathrm{K}$ antagonist (VKA) as the common comparator. 
The two primary efficacy endpoints were all-cause mortality and stroke or SE. The primary safety outcomes were the risk of major bleeding, intracranial bleeding and gastrointestinal bleeding. The study design was a network meta-analysis based on RCT. Two independent reviewers (SS and LN-F) searched MEDLINE/PubMed electronic databases using Cochrane highly sensitive search strategy for identifying randomised trials in MEDLINE: sensitivity-maximising version (2008 revision); PubMed format (supplemental material) and http://www. clinicaltrials.gov up to November 2015 to identify RCT comparing any treatment for stroke prevention in patients with AF. This included studies comparing VKA with placebo, APT (either single or dual) or NOAC. Also, all trials comparing LAAC with any percutaneous device against VKA were included. Keywords used for the search were "atrial fibrillation", "stroke", "stroke prophylaxis", "clinical trial”, "phase III”, "left atrial appendage closure", "non-vitamin K antagonist oral anticoagulants", "antiplatelet", "placebo" and "warfarin anticoagulation". The Preferred Reporting Items for Systematic Reviews and Meta-Analyses (PRISMA) statement for reporting systematic reviews and meta-analyses of RCT was used to extract data for this analysis. ${ }^{9}$ The quality of the studies was scored using a checklist for the assessment of the methodological quality. ${ }^{10}$

\section{Inclusion and exclusion criteria}

The trials included in the meta-analysis met all the following criteria: RCTs, articles in English language, inclusion of patients with non-valvular AF, reporting one of the following outcomes -all-cause mortality, incidence of stroke and SE, major or gastrointestinal bleeding and intracranial haemorrhage. Trials with fixed-dose warfarin, a combination of APT with NOAC or any other comparison without including VKA were excluded from the analysis. Trials involving therapy that were commercially withdrawn (eg. ximelagatran) were excluded. Any percutaneous device for LAAC was considered eligible, although only Watchman device trials met the inclusion criteria. Two independent authors (SS and LN-F) determined whether trials met inclusion criteria, with discrepancies being resolved by joint review and consensus. Trials with inadequate data to extract the above outcomes, trials without availability of full-text article and ongoing trials were also excluded.

\section{Statistical analysis}

Categorical variables were expressed as n (percentage) and continuous variables as mean (standard deviation) or median (interquartile range, IQR: $25-75$ th percentile). Data were analysed according to intention-to-treat analysis, when available. For each direct comparison between treatments, where information from more than one trial was available, we performed a traditional random-effect model. ${ }^{11}$ Q-statistic and Higgins' and Thompson's $\mathrm{I}^{2}$ test $^{12}$ were calculated to evaluate heterogeneity among the studies. At least moderate heterogeneity was considered to be present for $\mathrm{p}<0.10$ and an $\mathrm{I}^{2}>50 \%$. Publication bias was assessed by a funnel plot inspection and using the Begg and Egger's tests. ${ }^{13} 14$ For indirect comparisons, network metaanalysis was performed within a Bayesian framework computing HR and 95\% CIs with a random-effect hierarchical model by means of Markov chain Monte Carlo methods with Gibbs sampling from 1000 iterations obtained after a 5000 iteration training phase. Convergence was appraised graphically according to Gelman and Rubin. ${ }^{15}$ Statistical significance was accepted at the $95 \%$ confidence level $(p<0.05)$ and all data were analysed with the Stata Statistical Software: Release 14 (StataCorp. 2015; StataCorp, College Station, Texas, USA).

\section{RESULTS}

The flow diagram of the study analysis is shown in figure 1 . A total of 2929 citations were identified using the search terms. Of these, 488 were screened at the abstract level and 413 records were excluded. The remaining 75 publications were carefully screened and after analysis of the full text (see online supplementary file table S1), 19 studies met the prespecified inclusion criteria and were included in the network meta-analysis. ${ }^{6} 716-32$ The evidence network is shown in figure 2 before and after applying the inclusion and exclusion criteria. VKA were compared with NOAC in 6 studies, with APT in 10 studies, with LAAC with the Watchman device in 2 studies and with placebo in 3 studies. Of 87831 randomised patients, 36645 were assigned to warfarin, 43314 to NOACs, 6215 to APT, 925 to placebo and 732 patients were assigned to Watchman device implantation (table 1). Relevant features (baseline characteristics and outcomes) of included studies are listed in tables 1 and 2. The median follow-up was 21.6 months.

\section{Efficacy results}

All-cause mortality and stroke/SE incidence was reported in 16 and 17 studies, with 81316 and 86013 patients, respectively. Direct comparison revealed a mortality benefit with NOAC (odds ratio, OR 0.89 , CI 0.84 to 0.94 ) and no difference with LAAC (OR 0.68, CI 0.45 to 1.02 ) as compared with VKA. APT (OR 1.17, CI 0.97 to 1.40 ) and placebo (OR 1.75, CI 1.21 to 2.52) had worse mortality outcomes in comparison with warfarin (table 3, see online supplementary figure S1). While NOAC led to a significant reduction in the incidence of stroke or SE (OR 0.88, CI 0.81 to 0.95 ), LAAC led to a non-significant reduction (OR 0.84, CI 0.48 to 1.49 ) as compared with VKA. Both APT (OR 1.95, CI 1.59 to 2.40) and placebo (OR 3.54, CI 2.36 to 5.31) had higher risk of stroke in comparison with VKA (table 3, see online supplementary figure S2). The Begg $(\mathrm{Z}=0.53, \mathrm{p}=0.34)$ and Egger's test $(\mathrm{t}=1.72, \mathrm{p}=0.103)$ did not detect publication bias.

Indirect comparison revealed that LAAC was associated with lower rates of overall mortality than placebo (HR 0.38 ; CI 0.22 to $0.67 ; \mathrm{p}<0.001$ ) and APT (HR 0.58; CI 0.37 to 0.91 ; $\mathrm{p}=0.018)$. The mortality benefit of LAAC was not statistically significant in comparison with NOAC (HR 0.76; CI 0.50 to $1.16 ; p=0.211$ ) (figure 3A). Similarly, pooled HR of stroke or SE outcomes of LAAC with placebo (HR 0.24; CI 0.11 to 0.52 ; $\mathrm{p}<0.001$ ) and APT (HR 0.44; CI 0.23 to $0.86 ; \mathrm{p}=0.017$ ) showed a clear benefit in favour of LAAC. There was no difference in stroke or SE for LAAC compared with NOAC (HR 1.01 ; CI 0.53 to $1.92 ; \mathrm{p}=0.969$ ) (figure $3 \mathrm{~B}$ ).

\section{Safety results}

Major, intracranial and gastrointestinal bleedings were reported in 17 studies with 85,788, 85713 and 77116 patients, respectively. Direct comparison revealed a lower rate of major bleeding with NOAC (OR 0.77, CI 0.72 to 0.83 ) and placebo (OR 0.27, CI 0.12 to 0.63 ), and a non-significant difference with LAAC (OR 0.63, CI 0.33 to 1.19 ) and APT (OR 0.92, CI 0.74 to 1.14) as compared with warfarin (table 4, see online supplementary figure S3). Intracranial bleeding rate was reduced as compared with warfarin across all groups (table 4 , see online supplementary figure S4). Gastrointestinal bleeding was significantly higher with NOAC as compared with warfarin (OR 1.12, CI 1.01 to 1.25 ), but lower with the other groups as compared with warfarin (table 4, see online supplementary figure S5). 


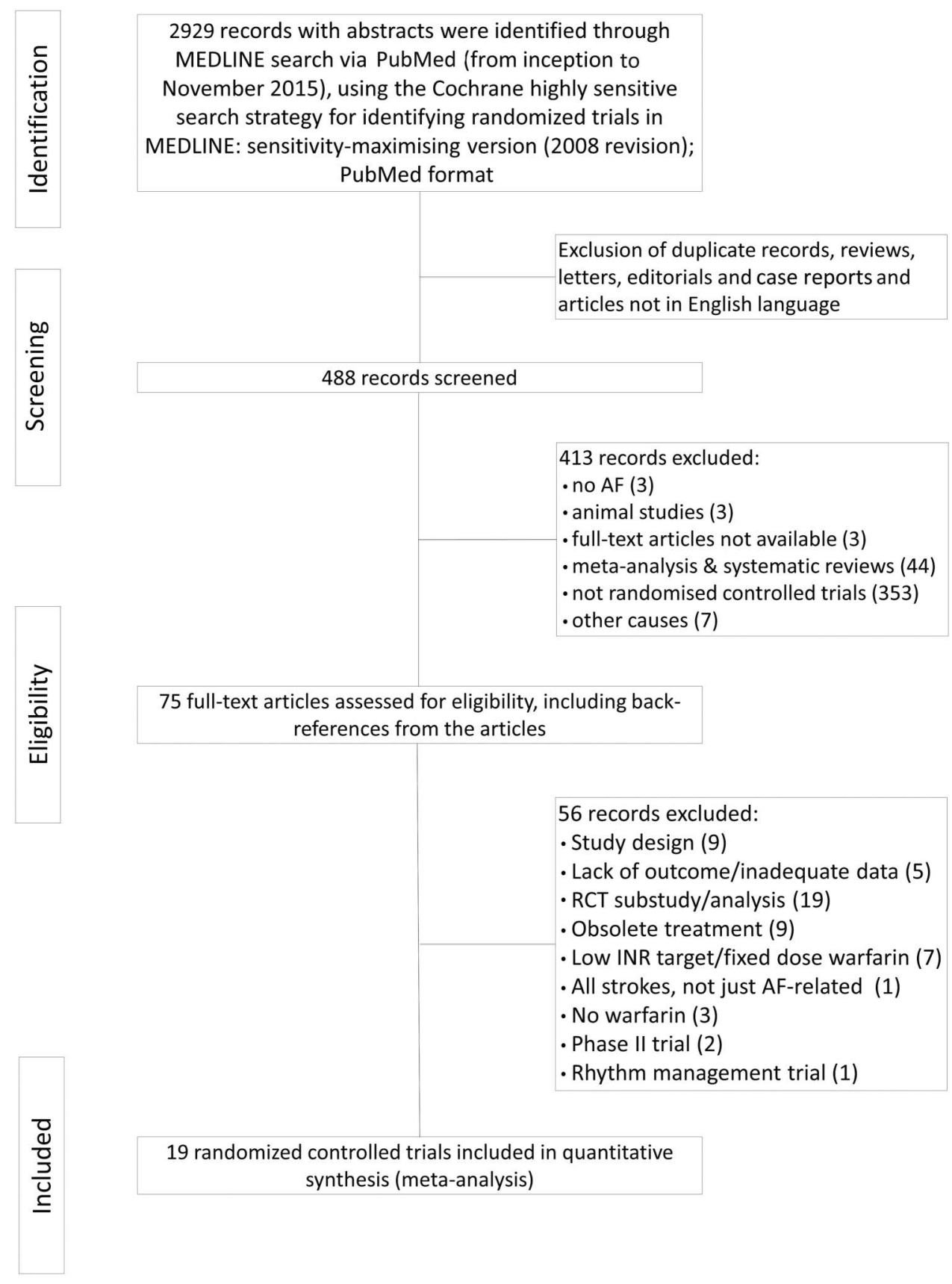

Figure 1 Flow chart of trials selection.

Figure 2 Network of antithrombotic treatment for patients with non-valvular atrial fibrillation with all searched studies (A) and network for selected studies and treatment comparisons for the meta-analysis (B). Dotted lines refer to indirect comparisons that have been performed.ASA, acetylsalicylic acid; APT, antiplatelet therapy; Fwarfarin, fixed dose of warfarin; DAPT, dual antiplatelet therapy; LAAC, left atrial appendage closure; NOAC, new oral anticoagulants; VKA, vitamin $\mathrm{K}$ antagonist.
2929 records with abstracts were identified through

MEDLINE search via PubMed (from inception to

ovember 2015), using the Cochrane highly sensitive

search strategy for identifying randomized trials in

EDLINE: sensitivity-maximising version (2008 revision)

PubMed format

- full-text articles not available (3)

- meta-analysis \& systematic reviews (44)

- not randomised controlled trials (353)

- other causes (7)

56 records excluded:

Study design (9)

uate data $(5)$

substudy/analysis (19)

- Low INR target/fixed dose warfarin (7)

(1)

No warfarin (3)

- Phase II trial (2

Rhythm management trial (1)

19 randomized controlled trials included in quantitative synthesis (meta-analysis) 
Table 1 Baseline clinical characteristics of the population from selected studies

\begin{tabular}{|c|c|c|c|c|c|c|c|c|c|c|c|c|}
\hline Trial & Year & Treatment & $\mathrm{N}$ & $\begin{array}{l}\text { Age } \\
(\text { mean } \pm S D), \\
\text { median (IQR) }\end{array}$ & Age $>75$ & Males & Hypertension & Diabetes & Heart failure & Stroke & $\mathrm{CHADS}_{2} \geq 2$ & Control \\
\hline \multicolumn{13}{|l|}{ LAAC trials } \\
\hline PREVAIL & 2014 & Watchman & 269 & $74.0 \pm 7.4$ & 140 & 182 & 238 & 91 & 63 & 74 & 248 & Warfarin \\
\hline PROTECT-AF & 2013 & Watchman & 463 & $71.7 \pm 8.8$ & 190 & 326 & 415 & 113 & 124 & 82 & 307 & Warfarin \\
\hline \multicolumn{13}{|l|}{ NOAC trials } \\
\hline ENGAGE-AF & 2013 & Edoxaban & 14069 & $72(64-78)$ & 5654 & 8670 & 13166 & 5103 & 8076 & 3982 & NA & Warfarin \\
\hline J-ROCKET-AF & 2012 & Rivaroxaban & 639 & $71(34-89)$ & 252 & 530 & 508 & 249 & 264 & NA & 639 & Warfarin \\
\hline ROCKET-AF & 2011 & Rivaroxaban & 7131 & $73(65-78)$ & NA & 4300 & 6436 & 2878 & 4467 & 3916 & 7130 & Warfarin \\
\hline ARISTOTLE & 2011 & Apixaban & 9120 & $70(63-76)$ & 2850 & 5986 & 7962 & 2284 & 3235 & 1748 & 6020 & Warfarin \\
\hline RELY & 2009 & Dabigatran & 12091 & $71.5 \pm 8.8$ & NA & 7705 & 9533 & 2811 & 3871 & 2428 & 8174 & Warfarin \\
\hline PETRO & 2007 & Dabigatran & 432 & $70.0 \pm 8.3$ & 139 & 352 & 307 & 111 & 123 & 74 & NA & Warfarin \\
\hline \multicolumn{13}{|l|}{ WARFARIN trials } \\
\hline PREVAIL & 2014 & Warfarin & 138 & $74.9 \pm 7.2$ & 78 & 103 & 134 & 41 & 32 & 39 & 126 & LAAC \\
\hline PROTECT-AF & 2013 & Warfarin & 244 & $72.7 \pm 9.2$ & 115 & 171 & 220 & 72 & 66 & 49 & 178 & LAAC \\
\hline ENGAGE-AF & 2013 & Warfarin & 7036 & 72 (64-78) & 2820 & 4575 & 6588 & 2521 & 4048 & 1991 & NA & Edoxaban \\
\hline J-ROCKET-AF & 2012 & Warfarin & 639 & $71.2(43-90)$ & 246 & 500 & 508 & 237 & 257 & NA & 639 & Rivaroxaban \\
\hline ROCKET-AF & 2011 & Warfarin & 7133 & $73(65-78)$ & NA & 4301 & 6474 & 2817 & 4441 & 3895 & 7131 & Rivaroxaban \\
\hline ARISTOTLE & 2011 & Warfarin & 9081 & $70(63-76)$ & 2828 & 5899 & 7954 & 2263 & 3216 & 1790 & 5998 & Apixaban \\
\hline RELY & 2009 & Warfarin & 6022 & $71.6 \pm 8.6$ & NA & 3809 & 4750 & 1410 & 1922 & 1195 & 4163 & Dabigatran \\
\hline PETRO & 2007 & Warfarin & 70 & $69.0 \pm 8.3$ & 19 & 59 & 49 & 15 & 24 & 13 & NA & Dabigatran \\
\hline BAFTA & 2007 & Warfarin & 488 & $81.5 \pm 4.3$ & 488 & 267 & 259 & 68 & 96 & 64 & 139 & Aspirin \\
\hline WASPO & 2007 & Warfarin & 36 & $83.5(80-90)$ & NA & 14 & 17 & 1 & 9 & NA & NA & Aspirin \\
\hline Vemmos et $a l^{24}$ & 2006 & Warfarin & 16 & $80.1 \pm 2.5$ & NA & 7 & 10 & 2 & 1 & NA & NA & Aspirin \\
\hline ACTIVE-W & 2006 & Warfarin & 3371 & $70.2 \pm 9.5$ & NA & 2211 & 2767 & 717 & 1040 & 510 & NA & DAPT \\
\hline WARSS & 2001 & Warfarin & 1103 & $63.3 \pm 11.2$ & NA & 656 & 746 & 367 & NA & 321 & NA & Aspirin \\
\hline PATAF & 1999 & Warfarin & 131 & 75.2 & NA & 58 & 46 & 25 & NA & NA & NA & Aspirin \\
\hline AFASAK II & 1999 & Warfarin & 170 & $74.2 \pm 7.7$ & NA & 97 & 80 & NA & NA & 9 & NA & Aspirin \\
\hline SPAF II & 1994 & Warfarin & 197 & $\begin{array}{l}64 \pm 8 \\
80 \pm 3\end{array}$ & NA & NA & NA & NA & NA & NA & NA & Aspirin \\
\hline EAFT & 1993 & Warfarin & 225 & $71.0 \pm 7.0$ & NA & 124 & 97 & 27 & 18 & NA & NA & Placebo/aspirin \\
\hline SPAF & 1991 & Warfarin & 210 & 67 & 17 & 155 & 103 & 25 & 29 & 17 & NA & Placebo \\
\hline AFASAK & 1989 & Warfarin & 335 & $72.8(41-88)$ & NA & 176 & 108 & 25 & 168 & 16 & NA & Placebo/aspirin \\
\hline \multicolumn{13}{|l|}{ ANTIPLATELET trials } \\
\hline BAFTA & 2007 & Aspirin & 485 & $81.5 \pm 4.2$ & 485 & 264 & 269 & 61 & 94 & 60 & 136 & Warfarin \\
\hline WASPO & 2007 & Aspirin & 39 & $82.6(80-90)$ & NA & 21 & 18 & 2 & 10 & NA & NA & Warfarin \\
\hline Vemmos et $\mathrm{al}^{24}$ & 2006 & Aspirin & 15 & $79.5 \pm 2.9$ & NA & 9 & 11 & 1 & 2 & NA & NA & Warfarin \\
\hline ACTIVE W & 2006 & DAPT & 3335 & $70.2 \pm 9.4$ & NA & 2219 & 2755 & 712 & 991 & 510 & NA & Warfarin \\
\hline WARSS & 2001 & Aspirin & 1103 & $62.6 \pm 11.4$ & NA & 653 & 753 & 338 & NA & 308 & NA & Warfarin \\
\hline PATAF & 1999 & Aspirin & 141 & 75.2 & NA & 67 & 53 & 21 & NA & NA & NA & Warfarin \\
\hline AFASAK II & 1999 & Aspirin & 169 & $73.1 \pm 7.2$ & NA & 110 & 73 & NA & NA & 9 & NA & Warfarin \\
\hline SPAF II & 1994 & Aspirin & 188 & & NA & NA & NA & NA & NA & NA & NA & Warfarin \\
\hline
\end{tabular}

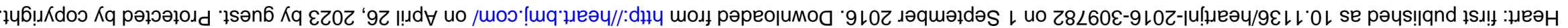




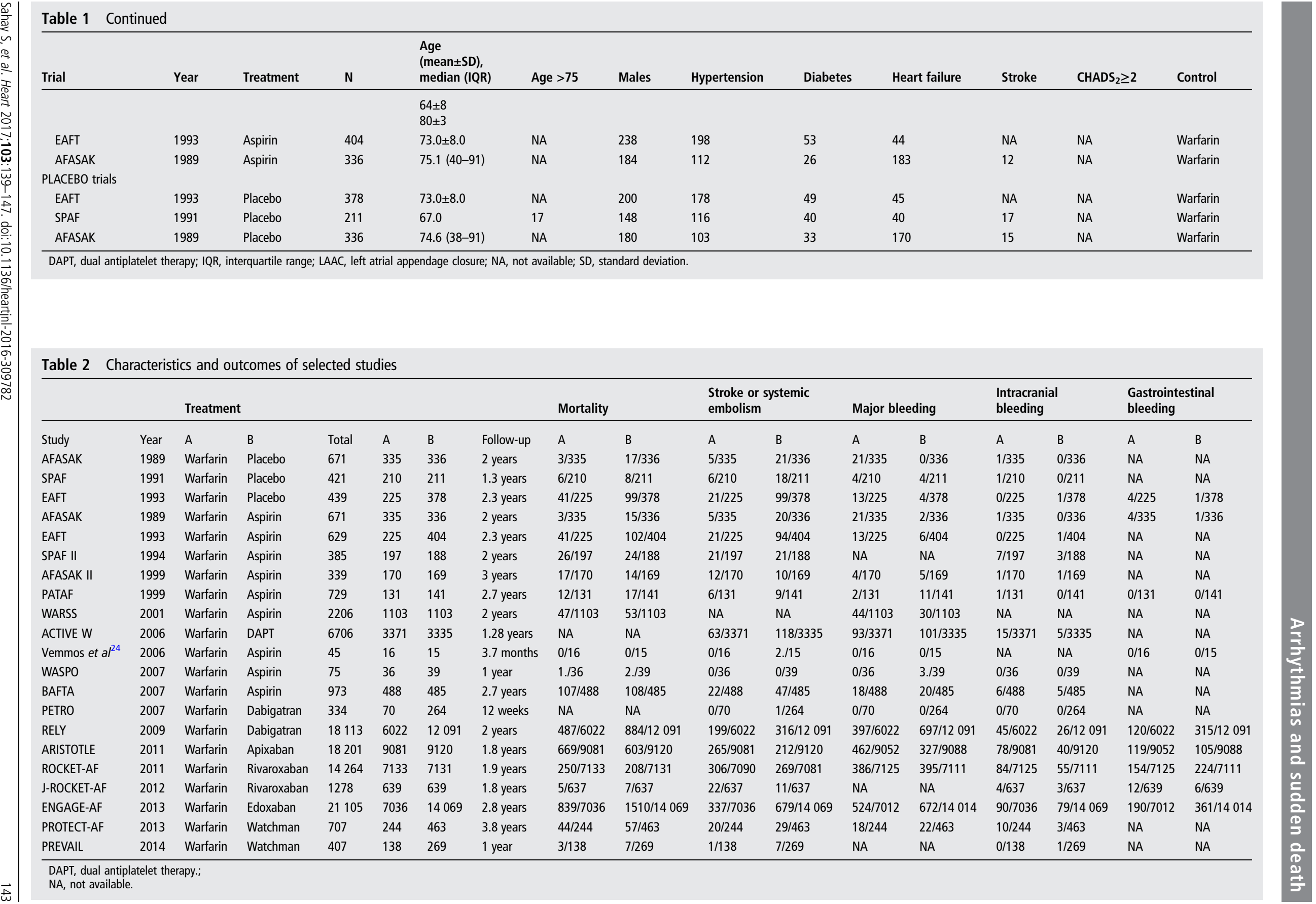

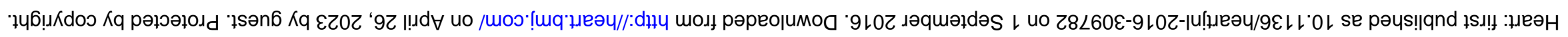


Table 3 Pooled efficacy outcomes with stroke prevention strategies directly compared with warfarin

\begin{tabular}{|c|c|c|c|c|}
\hline & Warfarin (events/n) & Comparator (events/n) & $\begin{array}{l}\text { Pairwise meta-analysis, } \\
\text { odds ratio }(95 \% \mathrm{Cl})\end{array}$ & $\begin{array}{l}\text { Heterogeneity, } \\
\mathrm{I}^{2}, \mathrm{p} \text { value }\end{array}$ \\
\hline \multicolumn{5}{|l|}{ All-cause mortality } \\
\hline Warfarin vs NOAC & $2250 / 29909$ & $3212 / 43048$ & 0.89 (0.84 to 0.94$)$ & $0.0 \%, p=0.881$ \\
\hline Warfarin vs LAAC & $47 / 382$ & $64 / 732$ & 0.68 (0.45 to 1.02$)$ & $0.0 \%, p=0.387$ \\
\hline Warfarin vs APT & $254 / 2685$ & $335 / 2865$ & 1.17 (0.97 to 1.40$)$ & $25.8 \%, p=0.223$ \\
\hline Warfarin vs placebo & $50 / 770$ & $124 / 925$ & 1.75 (1.21 to 2.52$)$ & $51.8 \%, p=0.126$ \\
\hline \multicolumn{5}{|c|}{ Stroke or systemic embolism } \\
\hline Warfarin vs NOAC & $1129 / 29936$ & $1488 / 43262$ & $0.88(0.81$ to 0.95$)$ & $45.7 \%, p=0.101$ \\
\hline Warfarin vs LAAC & $21 / 382$ & $36 / 732$ & $0.84(0.48$ to 1.49$)$ & $50.6 \%, p=0.155$ \\
\hline Warfarin vs APT & $150 / 4933$ & $321 / 5073$ & 1.95 (1.59 to 2.40$)$ & $47.4 \%, p=0.065$ \\
\hline Warfarin vs placebo & $32 / 770$ & $138 / 925$ & $3.54(2.36$ to 5.31$)$ & $0.0 \%, p=0.883$ \\
\hline
\end{tabular}

Indirect comparison for major bleeding with LAAC versus placebo (HR 2.33; CI 0.67 to $8.09 ; \mathrm{p}=0.183$ ), APT (HR 0.75 ; CI 0.30 to $1.88 ; \mathrm{p}=0.542$ ) and NOAC (HR 0.80 ; CI 0.33 to $1.94 ; \mathrm{p}=0.615)$ revealed no statistically significant differences (figure 4A). The risk of intracranial bleeding was not different between LAAC compared with APT (HR 0.42; CI 0.11 to 1.61; $\mathrm{p}=0.205$ ) or NOAC (HR $0.44 ;$ CI 0.13 to $1.49 ; \mathrm{p}=0.188$ ) (figure 4B). Gastrointestinal bleeding was significantly lower with LAAC compared with NOAC (HR 0.22; CI 0.09 to 0.56 ; $\mathrm{p}=0.001)$ and similar to placebo $(\mathrm{p}=0.563)$ and APT $(p=0.257)$ (figure 4C).

\section{DISCUSSION}

This network meta-analysis from RCTs showed that LAAC with the Watchman device is associated with higher survival and lower SE events compared with APT alone or placebo, with similar haemorrhagic outcomes. In comparison with NOAC, LAAC showed lower gastrointestinal bleeding risk with similar mortality and SE events.

Prevention of stroke in patients with AF is of high clinical relevance due to the disabling nature of stroke and associated high morbidity and mortality. Effective medical treatment consists of

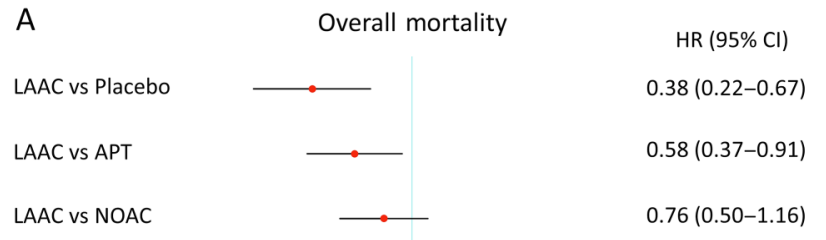

$$
\begin{array}{lll}
0,1 & \text { Favors LAAC } \quad 1 \text { Favors comparator } 10
\end{array}
$$

B

Stroke or systemic embolism

LAAC vs Placebo $\longrightarrow-10.11-0.52)$
LAAC vs APT

LAAC vs NOAC

$1.01(0.53-1.92)$
Figure 3 Pooled HR and 95\% Cls determined by network meta-analysis for efficacy outcomes: overall mortality $(A)$ and stroke or systemic embolism (B). anticoagulation, the benefits of which have to be weighed against potential bleeding complications. Given the fact that AF population is usually elderly and has other pre-existing comorbidities, bleeding events tend to complicate anticoagulation management. In fact, patients with higher stroke risk are usually those at the highest risk for bleeding. ${ }^{33}$ In clinical trials, around 20\% of the patients discontinue antithrombotic therapy. ${ }^{27-29}$ Eligible patients not receiving anticoagulation treatment are even higher in the real world. ${ }^{34}{ }^{35}$ LAAC has emerged as an alternative in patients with high-risk $\mathrm{AF}$ and contraindications to anticoagulation or high bleeding risk. ${ }^{8}$ On the other hand, LAAC is not exempt from periprocedural and midterm complications that may counterbalance its clinical benefit compared with placebo or APT. Given that direct comparisons by clinical trials between LAAC and placebo or APT are currently unavailable (and unlikely to be performed in the near future), we applied network meta-analysis methodology to perform this comparison. In fact, the effectiveness of LAAC as compared with no anticoagulation treatment is only based on theoretical risk reduction of ischaemic and bleeding events determined by scores (such as $\mathrm{CHA}_{2} \mathrm{DS}_{2}$-VASc and HAS-BLED) in registries. ${ }^{36-38}$ To the best of our knowledge, this is the first study to assess the efficacy and safety of LAAC compared with placebo or APT. Importantly, mortality and embolism events were significantly reduced with LAAC with no increase in safety events. These findings further support the evidence that LAAC may be recommended to patients deemed to be ineligible for oral anticoagulation. The rates of early ischaemic and bleeding complications in the two RCTs with LAAC were relatively high $^{39}$ compared with more recent registries. ${ }^{40}$ Thus, the benefit of LAAC may be even superior after overcoming the learning curve with increased operator experience and decreased periprocedural complications. However, these findings should be interpreted with caution, as various heterogeneous trials were included in the meta-analysis and direct comparison from original RCT should not be substituted by network meta-analysis. ${ }^{41}$

In addition, LAAC with the Watchman device has recently been approved in the USA by the Food and Drug Administration for clinical use as an alternative to warfarin for stroke prevention. ${ }^{5}$ The approval specific indications were in patients with non-valvular AF at increased risk of stroke or SE on the basis of $\mathrm{CHADS}_{2}$ or $\mathrm{CHA}_{2} \mathrm{DS}_{2}$-VASc and deemed to be eligible for anticoagulation therapy. In parallel, recent RCTs have shown that NOAC are an alternative to VKA with mostly better outcomes in terms of mortality, stroke and intracranial bleeding, with occasionally relatively higher risk of gastrointestinal bleeding. Thus, the use of NOAC is increasing, as they 
Table 4 Pooled safety outcomes with stroke prevention strategies directly compared with warfarin

\begin{tabular}{|c|c|c|c|c|}
\hline & Warfarin (events/n) & Comparator (events/n) & $\begin{array}{l}\text { Pairwise meta-analysis, } \\
\text { odds ratio }(95 \% \mathrm{Cl})\end{array}$ & $\begin{array}{l}\text { Heterogeneity, } \\
\mathrm{I}^{2}, \mathrm{p} \text { value }\end{array}$ \\
\hline \multicolumn{5}{|l|}{ Major bleeding } \\
\hline Warfarin vs NOAC & $1769 / 29211$ & $2091 / 42304$ & $0.77(0.72$ to 0.83$)$ & $90.9 \%, p=0.001$ \\
\hline Warfarin vs LAAC & $18 / 244$ & $22 / 463$ & $0.63(0.33$ to 1.19$)$ & - \\
\hline Warfarin vs APT & $195 / 5859$ & $178 / 6012$ & 0.92 (0.74 to 1.14$)$ & $74.6 \%, p=0.001$ \\
\hline Warfarin vs placebo & $38 / 770$ & $8 / 925$ & 0.27 (0.12 to 0.63$)$ & $71.4 \%, p=0.030$ \\
\hline \multicolumn{5}{|l|}{ Intracranial bleeding } \\
\hline Warfarin vs NOAC & $301 / 29901$ & $203 / 43028$ & $0.48(0.40$ to 0.57$)$ & $52.0 \%, p=0.080$ \\
\hline Warfarin vs LAAC & $10 / 382$ & $4 / 732$ & 0.21 (0.06 to 0.71$)$ & $41.9 \%, p=0.190$ \\
\hline Warfarin vs APT & $33 / 4917$ & $16 / 5058$ & $0.50(0.28$ to 0.92$)$ & $0.0 \%, p=0.908$ \\
\hline Warfarin vs placebo & $2 / 770$ & $1 / 925$ & $0.58(0.09$ to 3.70$)$ & $0.0 \%, p=0.701$ \\
\hline \multicolumn{5}{|l|}{ Gastrointestinal bleeding } \\
\hline Warfarin vs NOAC & $595 / 29850$ & $1011 / 42943$ & 1.12 (1.01 to 1.25$)$ & $77.9 \%, p=0.001$ \\
\hline Warfarin vs LAAC & $21 / 382$ & $3 / 722$ & $0.24(0.11$ to 0.51$)$ & - \\
\hline Warfarin vs APT & 9/868 & $7 / 1065$ & $0.56(0.18$ to 1.76$)$ & $53.9 \%, p=0.114$ \\
\hline Warfarin vs placebo & $8 / 560$ & $1 / 714$ & $0.13(0.02$ to 0.76$)$ & $0.0 \%, p=0.876$ \\
\hline
\end{tabular}

A

Major bleeding

$\mathrm{HR}(95 \% \mathrm{Cl})$

LAAC vs Placebo

LAAC vs APT

LAAC vs NOAC

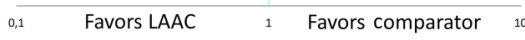

B

Intracranial bleeding

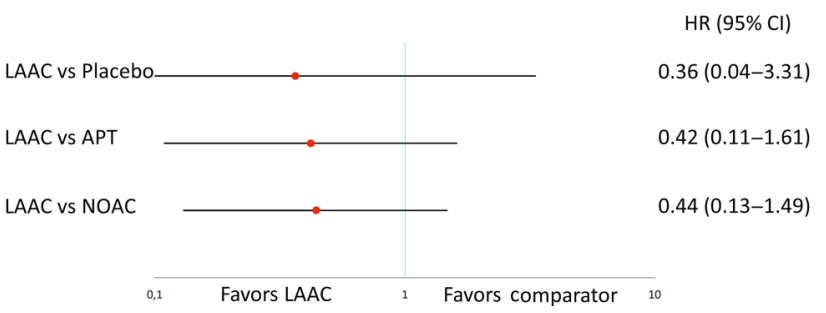

C

Gastrointestinal bleeding

$\operatorname{HR}(95 \% \mathrm{CI})$

LAAC vs Placebo

LAAC vs APT

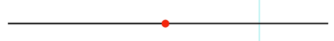

LAAC vS NOAC

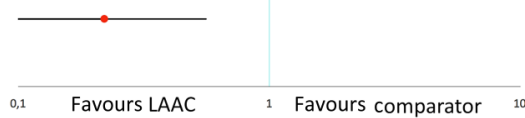

Figure 4 Pooled HR and 95\% Cls determined by network meta-analysis for safety outcomes: major bleeding $(A)$, intracranial bleeding (B) and gastrointestinal bleeding (C).

mitigate some disadvantages of VKA with better clinical outcomes. However, any type of anticoagulation still confers a permanent risk of bleeding, as compared with placebo, and NOAC are not indicated in patients with severe renal dysfunction. Furthermore, in clinical trials ${ }^{27-29}$ and real-life registries, ${ }^{35} 42$ there is a $13-25 \%$ discontinuation rate per 100 patient-year. The main reason for discontinuation was bleeding events, followed by NOAC side effects. Thus, in the absence of head-to-head comparison between NOAC and LAAC (and unlikely to have data in near future), it is clinically relevant to determine the clinical effects of both treatments. In accordance with previous studies, ${ }^{43} 44$ our network meta-analysis demonstrated by indirect comparison that there was no significant difference between LAAC and NOAC in terms of mortality and stroke or SE prevention. Gastrointestinal bleeding was significantly lower with LAAC, with similar overall major bleeding rates. Although intracranial bleeding rate between LAAC compared with NOAC was not statistically different in our study, this was probably related to the small sample size in the LAAC arm and limited follow-up. Interestingly, Koifman et $a l^{43}$ found a lower risk of haemorrhagic stroke with the Watchman device when including non-randomised trials in the analysis. Also, as anticoagulation confers $\sim 2 \%$ annual risk of major bleeding, the differences may have been more evident with a longer follow-up period. Another issue when evaluating new therapies such as NOAC and LACC is cost. A recent study showed that both treatments were cost-effective relative to warfarin, but LAAC was found to offer a better value relative to NOAC. ${ }^{45}$ On the contrary, Micieli et $a l^{46}$ found that apixaban is the most costeffective therapy in patients with AF compared with other NOAC and LAAC. Future studies are needed to clarify the benefits and the cost-effectiveness of LAAC compared with NOAC.

\section{Limitations}

This study, as with any meta-analysis, has the limitations of the original studies. Heterogeneity of the study designs and patient profile was present. Network meta-analysis assumes that patients enrolled in the different studies would have been sampled from the same theoretical population and, using VKA patients as bridge comparators, the indirect comparison may be biased. Moreover, we could not test inconsistencies between direct and indirect comparison, as no trials compare LAAC versus other medical treatment besides VKA. All patients with LAAC are treated with anticoagulants for the initial 45 days, dual APT for 6 months and single APT thereafter, which is a confounding factor when estimating the bleeding risk. However, this study 
assesses the LAAC bleeding risk taking into account the LAAC plus the protocol-mandatory antithrombotic therapy, as a single intervention. Approximately, 95\% of patients can be withdrawn from anticoagulation treatment after a follow-up transoesophageal echo at 6 weeks. There were only two RCTs comparing LAAC with VKA, and warfarin showed an unexpected low rate of stroke in one of them. ${ }^{7}$ The relatively small sample size and the very low event rates of gastrointestinal and intracranial bleeding, with wide CIs, might underpower the comparison making it difficult to interpret the data. Finally, we have included some studies with low-dose warfarin, which can bias the safety as well as efficacy outcomes.

\section{CONCLUSION}

NOAC and LAAC are currently the two therapeutic strategies with the best efficacy and safety profile for embolism prevention in patients with non-valvular AF. LAAC obviates the need for life-long anticoagulation and the findings of this meta-analysis suggest that LAAC is associated with better efficacy and similar safety profiles, respectively, compared with placebo or APT. By indirect comparison, there seems to be no significant difference between LAAC and NOAC in terms of mortality and stroke/SE prevention, with similar major bleeding risk, making it a potent alternative to medical prophylaxis in patients with AF. Future studies will have to confirm these results.

\section{Key messages}

What is already known on this subject?

Vitamin $\mathrm{K}$ antagonists (VKA) are highly effective in patients with atrial fibrillation (AF) for stroke prevention, but its use is limited by drug interactions, narrow therapeutic range and increased bleeding risk. Non-vitamin K antagonist oral anticoagulants (NOAC) continue to have an increased risk of major bleeding as compared with placebo. Anticoagulation is frequently interrupted in a high proportion ( 20\%) of patients with $\mathrm{AF}$ with previous or high risk of bleeding, and antiplatelet therapy (APT) or no treatment is generally advised in this situation. Left atrial appendage closure (LAAC) has recently emerged as an effective treatment for stroke prevention in patients with AF. However, comparisons between LAAC versus medical treatment other than VKA are lacking.

\section{What might this study add?}

This network meta-analysis assessed the efficacy and safety of LAAC compared with medical therapy (NOAC, APT and placebo) for stroke prevention in patients with non-valvular AF. Importantly, LAAC was associated with higher survival and lower systemic embolism (SE) events compared with APT alone or placebo, with similar bleeding outcomes. In comparison with NOAC, LAAC showed lower gastrointestinal bleeding, with similar SE events.

\section{How might this impact on clinical practice?}

The results of this meta-analysis provide further insight into the clinical efficacy and safety of LAAC, which may be considered as a stroke prevention therapy in patients deemed to be ineligible for oral anticoagulation on long-term basis. This study could contribute to improving the treatment planning in this common group of patients.
Contributors SS and LN-F: (1) conception and design, and interpretation of data; (2) drafting and revising of the manuscript; and (3) final approval of the manuscript submitted. JR-C, PJ-Q, PS, CB, IN-G, NG, JAdA, MdT, LPdl, AF-O, JE, CM: (1) critical review of the manuscript for important intellectual content and (2) final approval of the manuscript submitted.

Funding Relationship with industry: JR-C has received a research grant from Edwards Lifesciences, St Jude Medical and Medtronic. All other authors have reported that they have no relationships relevant to the contents of this paper to disclose.

Competing interests None declared.

Provenance and peer review Not commissioned; externally peer reviewed.

\section{REFERENCES}

1 Lip GY, Tse HF, Lane DA. Atrial fibrillation. Lancet 2012;379:648-61.

2 Lip GY, Lane DA. Stroke prevention in atrial fibrillation: a systematic review. JAMA 2015;313:1950-62.

3 January CT, Wann LS, Alpert JS, et al. 2014 AHA/ACC/HRS guideline for the management of patients with atrial fibrillation: a report of the American College of Cardiology/American Heart Association Task Force on practice guidelines and the Heart Rhythm Society. Circulation 2014;130:e199-267.

4 Ruff CT, Giugliano RP, Braunwald E, et al. Comparison of the efficacy and safety of new oral anticoagulants with warfarin in patients with atrial fibrillation: a meta-analysis of randomised trials. Lancet 2014;383:955-62.

5 Maisel WH. FDA Approval for Watchman Device. 13 March 2015. http://www. accessdata.fda.gov/cdrh_docs/pdf13/P130013a.pdf (accessed 20 Mar 2016).

6 Reddy VY, Doshi SK, Sievert H, et al. Percutaneous left atrial appendage closure for stroke prophylaxis in patients with atrial fibrillation: 2.3-Year Follow-up of the PROTECT AF (Watchman Left Atrial Appendage System for Embolic Protection in Patients with Atrial Fibrillation) Trial. Circulation 2013;127:720-9.

7 Holmes DR Jr, Kar S, Price MJ, et al. Prospective randomized evaluation of the Watchman Left Atrial Appendage Closure device in patients with atrial fibrillation versus long-term warfarin therapy: the PREVAIL trial. J Am Coll Cardiol 2014;64:1-12.

8 CammAJ, Lip GY, De Caterina R, et al. 2012 focused update of the ESC Guidelines for the management of atrial fibrillation: an update of the 2010 ESC Guidelines for the management of atrial fibrillation. Developed with the special contribution of the European Heart Rhythm Association. Eur Heart J 2012;33:2719-47.

9 Liberati A, Altman DG, Tetzlaff J, et al. The PRISMA statement for reporting systematic reviews and meta-analyses of studies that evaluate health care interventions: explanation and elaboration. PLoS Med 2009;6:e1000100.

10 Downs SH, Black N. The feasibility of creating a checklist for the assessment of the methodological quality both of randomised and non-randomised studies of health care interventions. J Epidemiol Community Health 1998;52:377-84.

11 DerSimonian R, Laird N. Meta-analysis in clinical trials. Clin Trials 1986;7:177-88.

12 Higgins JP, Thompson SG. Quantifying heterogeneity in a meta-analysis. Stat Med 2002;21:1539-58.

13 Begg CB, Mazumdar M. Operating characteristics of a rank correlation test for publication bias. Biometrics 1994;50:1088-101.

14 Egger M, Davey Smith G, Schneider M, et al. Bias in meta-analysis detected by a simple, graphical test. BMJ 1997;315:629-34.

15 Gelman A, Rubin DB. Markov chain Monte Carlo methods in biostatistics. Stat Methods Med Res 1996;5:339-55.

16 Cohen BA, Feldman G, Flaker GC, et al. Stroke prevention in atrial fibrillation study. Final results. Circulation 1991;84:527-39.

17 Petersen P, Boysen G, Godtfredsen J, et al. Placebo-controlled, randomised trial of warfarin and aspirin for prevention of thromboembolic complications in chronic atrial fibrillation. The Copenhagen AFASAK Study. Lancet 1989;1:175-9.

18 Koudstaal PJ, van Gijn J, Bogousslavsky J, et al. Secondary prevention in non-rheumatic atrial fibrillation after transient ischaemic attack or minor stroke. EAFT (European Atrial Fibrillation Trial) Study Group. Lancet 1993;342:1255-62.

19 McBride R, Halperin JL, Hart RG, et al. Warfarin versus aspirin for prevention of thromboembolism in atrial fibrillation: Stroke Prevention in Atrial Fibrillation II Study. Lancet 1994;343:687-91.

20 Rash A, Downes T, Portner $\mathrm{R}$, et al. A randomized controlled trial of warfarin versus aspirin for stroke prevention in octogenarians with atrial fibrillation (WASPO). Age Ageing 2007;36:151-6.

21 Hankey GJ. Warfarin-Aspirin Recurrent Stroke Study (WARSS) trial: is warfarin really a reasonable therapeutic alternative to aspirin for preventing recurrent noncardioembolic ischemic stroke? Stroke 2002;33:1723-6.

22 Mant J, Hobbs FD, Fletcher $\mathrm{K}$, et al. Warfarin versus aspirin for stroke prevention in an elderly community population with atrial fibrillation (the Birmingham Atrial Fibrillation Treatment of the Aged Study, BAFTA): a randomized controlled trial. Lancet 2007;370:493-503.

23 Gullov AL, Koefoed BG, Petersen P. Bleeding during warfarin and aspirin therapy in patients with atrial fibrillation. The AFASAK 2 study. Arch Intern Med 1999;159:1322-8. 
24 Vemmos KN, Tsivgoulis G, Spengos K, et al. Primary prevention of arterial thromboembolism in the oldest old with atrial fibrillation-a randomized pilot trial comparing adjusted-dose and fixed low-dose coumadin with aspirin. Eur J Intern Med 2006;17:48-52.

25 Connolly S, Pogue J, Hart R, et al. Clopidogrel plus aspirin versus oral anticoagulation for atrial fibrillation in the Atrial fibrillation Clopidogrel Trial with Irbesartan for prevention of Vascular Events (ACTIVE W): a randomized controlled trial. Lancet 2006;367:1903-12.

26 Hellemons BSP, Langenberg $M$, Lodder J, et al. Primary prevention of arterial thromboembolism in non-rheumatic atrial fibrillation in primary care: randomized controlled trial comparing two intensities of coumarin with aspirin. BMJ 1999:319:958-64.

27 Connolly SJ, Ezekowitz MD, Yusuf S, et al. Dabigatran versus warfarin in patients with atrial fibrillation. N Eng J Med 2009;361:1139-51.

28 Patel MR, Mahaffey KW, Garg J, et al. Rivaroxaban versus warfarin in nonvalvular atrial fibrillation. N Eng J Med 2011;365:883-91.

29 Granger CB, Alexander JH, McMurray JJV, et al. Apixaban versus warfarin in patients with atrial fibrillation. N Eng J Med 2011;365:981-92.

30 Giugliano RP, Ruff CT, Braunwald E, et al. Edoxaban versus warfarin in patients with atrial fibrillation. N Eng J Med 2013;369:2093-104.

31 Hori M, Matsumoto M, Tanahashi N, et al. Rivaroxaban versus warfarin in Japanese patients with atrial fibrillation-the J-ROCKET AF study. Circ J 2012;76:2104-11.

32 Ezekowitz MD, Reilly PA, Nehmiz G, et al. Dabigatran with or without concomitant aspirin compared with warfarin alone in patients with nonvalvular atrial fibrillation (PETRO Study). Am J Cardiol 2007;100:1419-26.

33 Friberg L, Rosenqvist M, Lip GY. Evaluation of risk stratification schemes for ischaemic stroke and bleeding in 182,678 patients with atrial fibrillation: the Swedish Atrial Fibrillation cohort study. Eur Heart J 2012;33:1500-10.

34 Nieuwlaat R, Capucci A, Camm AJ, et al. Atrial fibrillation management: a prospective survey in ESC member countries: the Euro Heart Survey on Atrial Fibrillation. Eur Heart J 2005;26:2422-34.

35 Beyer-Westendorf J, Ebertz F, Förster K, et al. Effectiveness and safety of dabigatran therapy in daily-care patients with atrial fibrillation: results from the Dresden NOAC Registry. Thromb Haemost 2015;113:1247-57.

36 Urena M, Rodés-Cabau J, Freixa X, et al. Percutaneous left atrial appendage closure with the AMPLATZER cardiac plug device in patients with nonvalvular atrial fibrillation and contraindications to anticoagulation therapy. J Am Coll Cardiol 2013;62:96-102.

37 López Mingle JR, Asensio JM, Gragera JE, et al. Two-year clinical outcome from the Iberian registry patients after left atrial appendage closure. Heart 2015; 101:877-83.

38 Tzikas A, Shakir S, Gafoor S, et al. Left atrial appendage occlusion for stroke prevention in atrial fibrillation: multicenter experience with the AMPLATZER Cardiac Plug. Eurolntervention 2016;11:1170-9.

39 Holmes DR Jr, Doshi SK, Kar S, et al. Left atrial appendage closure as an alternative to warfarin for stroke prevention in atrial fibrillation: a patient-level meta-analysis. J Am Coll Cardiol 2015;65:2614-23.

40 Boersma LV, Schmidt B, Betts TR, et al. Implant success and safety of left atrial appendage closure with the WATCHMAN device: peri-procedural outcomes from the EWOLUTION registry. Eur Heart J 2016. Published Online First: 27 Jan 2016. http:// dx.doi.org/10.1093/eurheartj/ehv730

41 Skjøth F, Larsen TB, Rasmussen LH. Indirect comparison studies-are they useful? Insights from the novel oral anticoagulants for stroke prevention in atrial fibrillation. Thromb Haemost 2012;108:405-6.

42 Al-Khalili F, Lindström C, Benson L. The safety and persistence of non-vitamin-K-antagonist oral anticoagulants in atrial fibrillation patients treated in a well structured atrial fibrillation clinic. Curr Med Res Opin 2016;32: 779-85.

43 Koifman E, Lipinski MJ, Escarcega RO, et al. Comparison of Watchman device with new oral anti-coagulants in patients with atrial fibrillation: a network meta-analysis. Int J Cardiol 2016;205:17-22.

44 Li X, Wen SN, Li SN, et al. Over 1-year efficacy and safety of left atrial appendage occlusion versus novel oral anticoagulants for stroke prevention in atrial fibrillation: a systematic review and meta-analysis of randomized controlled trials and observational studies. Heart Rhythm 2016;13:1203-14.

45 Reddy VY, Akehurst RL, Armstrong SO, et al. Time to cost-effectiveness following stroke reduction strategies in AF. J Am Coll Cardiol 2015;66: 2728-39.

46 Micieli A, Wijeysundera HC, Qiu F, et al. A decision analysis of percutaneous left atrial appendage occlusion relative to novel and traditional oral anticoagulation for stroke prevention in patients with new-onset atrial fibrillation. Med Decis Making 2016;36:366-74 